\title{
ANALYSIS OF DATA TRANSMISSION IN WIRELESS-LAN FOR 802.11
}

\section{E2ET}

\author{
Boddu Manikanta ${ }^{1}$, Chuppana Sampath Kumar ${ }^{2}$ \\ ${ }^{1}$ Student, ${ }^{2}$ Associate Professor, Department of ECE, Chaitanya engineering college, Andhra Pradesh \\ maninani.ashok@gmail.com,amorous_sam@yahoo.co.in
}

\begin{abstract}
The aim of this paper is to investigate the transmission of data between client and server through different IEEE 802.11 wireless LAN multi- HOP network. To improve this issue, the transmission opportunity (TXOP) mechanism is defined in the IEEE 802.11e standard, with which a wireless node can transmit multiple frames consecutively for a maximum channel occupancy time, called TXOP limit. This paper considers the performance of the TXOP mechanism for multi-hop wireless networks. Focusing on a three-node chain topology, we model it as a tandem queuing network with two nodes. The E2ET is derived and the analysis is validated by simulation. Numerical results show that the TXOP mechanism works well for multi-hop wireless networks. It is also shown that adjusting TXOP limit is significantly important in order to increase the overall throughput. In terms of multi-hop wireless networks, there is little analytical work with regard to the E2ET performance. One of the rationales for the analytical difficulty for multi-hop wireless networks is that IEEE 802.11 MAC protocol is too complex to model the behavior of multi-hop frame transmissions.
\end{abstract}

Keywords: IEEE 802.11e, multi-hop wireless LAN, TXOP, E2ET

\section{INTRODUCTION}

IEEE 802.11-based multihop wireless net-works have attracted much interest for next-generation networks [1]. The distributed coordination function (DCF) is a fundamental mechanism of the medium access control (MAC) protocol for IEEE 802.11, employing Carrier Sense Multiple Access with Collision Avoidance (CSMA/CA). The IEEE 802.11 DCF specifies random back off algorithm, with which each wireless node DCF transmits data in an autonomously distributed manner. It can provide a high-speed wireless connection (e.g. IEEE802.11g provides up to $54 \mathrm{Mb} / \mathrm{s}$ data rate), however, it is well known that the effective end-to-end throughput (E2ET) degrades due to the overhead caused by the random back off and the four-way handshake mechanism based on the exchange of Request-To-Send/Clear-To-Send (RTS/CTS) frames. To improve this issue, the transmission opportunity (TXOP) mechanism is defined in IEEE 802.11e, enabling a wireless node to transmit multiple frames consecutively for a maximum channel occupancy time called TXOP limit [2]. The TXOP mechanism is expected to enhance significantly the capacity in order to accommodate multimedia traffic such as video streaming and Voice over Internet Protocol (VoIP).

It is well known that in multi-hop wireless backhaul net-works with homogeneous frame flows, the E2ET of a frame flow degrades significantly with the increase in the number of its transmission hops. Since the TXOP mechanism was developed for single-hop communication, it greatly improves the throughput for single-hop communication. However, it is not clear how the E2ET in multihop wireless networks is improved by the TXOP mechanism. The aim of this paper is to develop an analytical model with which the sensitivity of the TXOP limit to the E2ET can be quantitatively investigated.

In this, we consider how the TXOP mechanism improves the E2ET in a backhaul-type wireless mesh network. Our analysis consists of two steps. First, focusing on a three-node chain topology, we model it as a tandem queuing network with two nodes. With this model, we analyze the joint distribution of the numbers of frames in the two nodes and the server state, deriving the E2ET. Note that in the tandem queuing network model, we simply assume that the time intervals caused by the back off mechanism are exponentially distributed, depending on which node completes the last frame transmission [11]. In the second step, we analyze the back off-timer process observed at the completion epoch of a frame transmission. Using the analytical results of the second step, we can determine the parameters of exponential distributions for time intervals caused by the back off mechanism. We validate the analysis with simulation.

\section{LITERATURE OF MULTI-HOP}

There is much literature on the performance of TXOP mechanism for multihop wireless networks (see, e.g., [3]-[6]), however, most of the previous studies are based on simulation, and there is little analytical work taking into account the flowlevel behavior resulting from the IEEE 802.11 MAC protocol. 
In terms of single-hop wireless networks, [8] proposed an analytical model for TXOP mechanism in WLANs under various channel conditions. They modeled the transmission queue of each node by a two-state continuous-time Markov chain which captures the burst transmission mechanism and the wireless channel errors. By using their analytical model, they could obtain the performance metrics including the throughput and buffer overflow probability. [9] also proposed an analytical model to evaluate the performance of TXOP mechanism for single-hop networks. They divided the transmission duration into three different components, successful transmit overhead, collision time overhead and data transmission. They specified the values of these components for two different access modes: basic access and RTS/CTS access, showing how to calculate the total throughput achieved under each access mode. Lee et al. [10] introduced a three dimensional Markov chain model to evaluate the throughput for IEEE 802.11e under saturation condition. Their model captured IEEE 802.11e parameters, and a numerical approach was proposed to obtain the set of parameters to meet the performance requirements.

In terms of multihop wireless networks, there is little analytical work with regard to the E2ET performance. One of the rationales for the analytical difficulty for multihop wireless networks is that IEEE 802.11 MAC protocol is too complex to model the behavior of multihop frame transmissions.[11] derived the maximum E2ET of multi-hop networks, taking location-dependant neighboring interference into consideration. A strong point of their approach is that the maximum E2ET of any pair of nodes in a multi-hop network can be calculated. However, the impact of parameters such as the offered load and buffer size of a wireless node on the E2ET cannot be investigated. In our three-node tandem network model, on the other hand, location-dependant neighboring interference is not taken into consideration. However, the three-node tandem network model enables us to investigate the sensitivity of the parameters including TXOP limit to the E2ET.

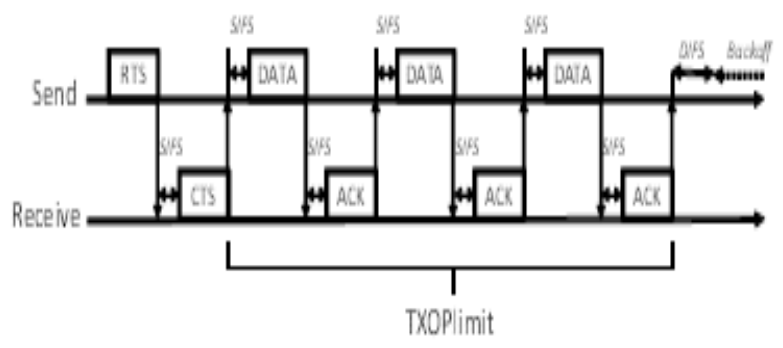

Fig. 1 Transmission opportunity (TXOP) mechanism

\section{TRANSMISSION OPPORTUNITY (TXOP) MECHANISM}

IEEE 802.11e standard has been issued to introduce quality of service (QoS) support for wireless LANs. The Enhanced Distributed Channel Access (EDCA) is used as the fundamental access mechanism for the MAC layer in IEEE 802.11e. In the EDCA, relative priority service is supported in order to improve the multimedia transmission such as VoIP, and the TXOP is a principal mechanism of the EDCA to guarantee QoS required by real-time applications. To reduce the overhead of the contention time, the TXOP allows multiple consecutive frame exchanges without back off.

Fig. 1 shows the frame-transmission diagram of three data frames by using the TXOP mechanism. In the TXOP transmission, a sender node can transmit multiple frames within a pre specified time interval called TXOP limit. After a frame transmission, the next frame is transmitted immediately after a successful ACK reception and the Short Inter Frame Space (SIFS). If the sender node cannot receive the ACK frame, it terminates the TXOP transmission and sends frames again after another back off interval. When the TXO Limit is over, the node goes into back off phase.

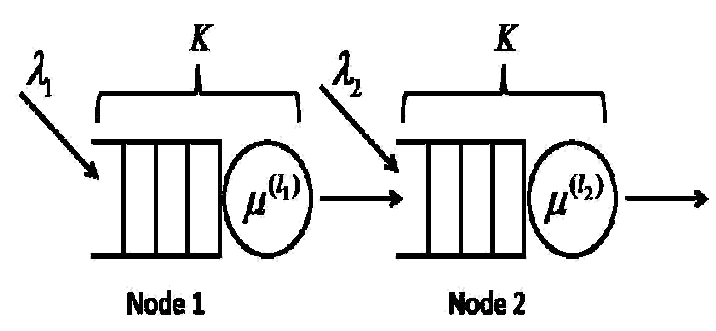

Fig. 2 Queuing model

Using TXOP mechanism, data transfer efficiency is im-proved since the frames included in the same burst share the overhead per transmission opportunity. Hence, the TXOP mechanism provides high throughput performance and low delay for congested networks.

\section{DATA TRANSMISSION BETWEEN CLIENT AND SREVER}

We consider a wireless network with three-node chain topology, which consists of Nodes 1 and 2 and the gateway $(\mathrm{GW})$. Node 1 and $\mathrm{GW}$ are out of the transmission range but within the sensing range of each other, while each pair of neighboring nodes among the three nodes is within the transmission range. We assume that a single frequency channel is used for frame transmission, which implies that any two nodes cannot use the channel simultaneously. In the wireless network, Flows 1 and 2 are transmitted. Flow 1 is 
transmitted from Node 1 to GW via Node 2, and Flow 2 is from Node 2 to GW. Nodes 1 and 2 use burst transmission mechanism, and a node having the transmission right sends frames continuously during TXOP limit.

We model the wireless network as a tandem queuing network with two nodes. (See Fig. 2.) We assume that frames arrive at Node 1 (resp. 2) according to a Poisson process with rate 1 (resp. 2). The system capacity of each node is equal to (frames). After the transmission right is assigned to Node 1 (resp. 2), Node 1 (resp. 2) sends at most 1 (resp. 2) frames during the TXOP limit. Frames transmitted by Node 1 join the queue of Node 2, while those transmitted by Node 2 leave the network. Either one but not both of the two nodes can transmit a frame when it gets ready for transmission. When the frame transmission of Node 1 (resp. 2) completes, Node 1 or 2 gets ready for frame transmission after a time interval consisting of DIFS and the back off time. Such interval of Node 1 (resp. 2).

The data transmission takes place with server and client between nodes analysis. The last assumption (called the inactive-period assumption hereafter) implies that when one of the two nodes completes a frame transmission, each node is forced to be inactive for an exponential time interval. Note that a node in inactive state cannot transmit a frame even when it has the frame in the buffer. If the inactive period of the node is over and the node has no frames to transmit, then the idle period of the node starts. Further when the node is in idle state and a new frame arrives at the node, the node immediately starts the frame transmission.

\section{THROUGHPUT ANALYSIS}

The value of the TXOP limit affects the throughput performance. Fig. 6 represents E2ETs of Flows 1 and 2 against TXOP limit 1 and 2. In this figure, the offered loads of both flows are the same and equal to $8 \mathrm{Mb} / \mathrm{s}$ and $12 \mathrm{Mb} / \mathrm{s}$. From the fact, we can improve the throughput performance for multihop wireless networks to use TXOP mechanism. However, for 1, $2>20$, all the E2ETs in the figure remain almost constant. Using TXOP mechanism for multihop wireless networks has both positive and negative effects. The positive effect is the reduction of the overhead caused by the back off algorithm and by the exchange of control frames. This enables wireless nodes to send data frames efficiently. On the other hand, the negative effect is that the node having the transmission right is likely to use the network channel during a large period of time. Occasionally, this channel occupation leads to deteriorate the performance in multihop wireless networks. In our model, if Node 1 occupies the network channel for a long term and sends lots of frames at a time, the Node 2's queue is overflowed, degrading the throughput performance significantly. End-to-end throughput (E2EPT) transmission takes place with overload of data transmission.

\section{SIMULATION RESULTS}

The simulation result explains about the nodes design from client to server.

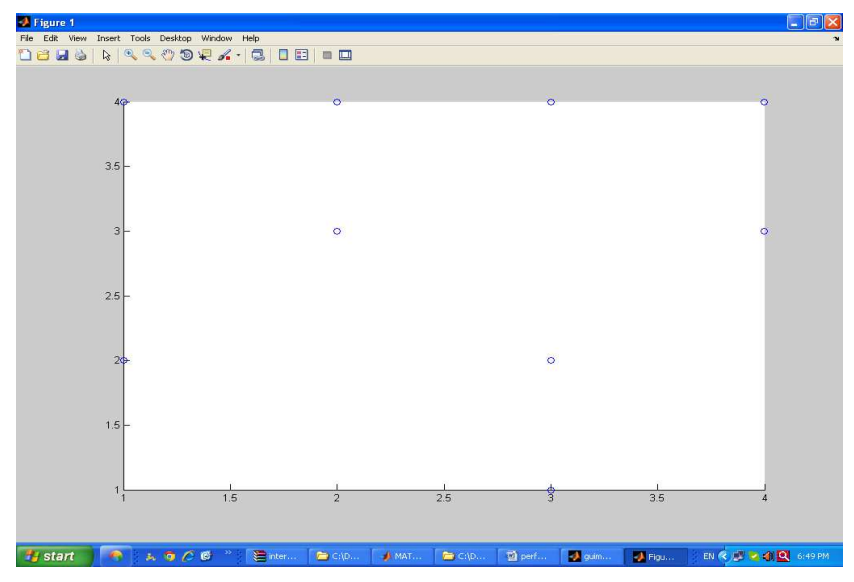

Fig- 3 Client to server Nodes design

The result explains about the message information transfer from client to server from the nodes.

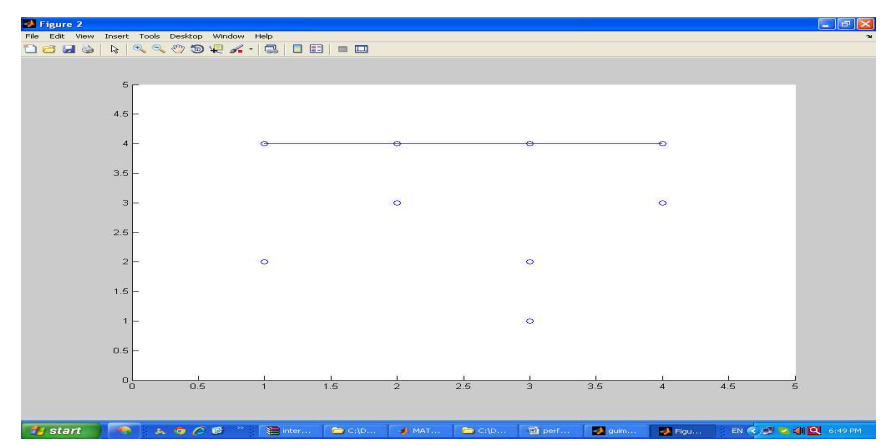

Fig-4 Path design from client to server

The plot explains about the acknowledgment received from the message transfer from client to server.

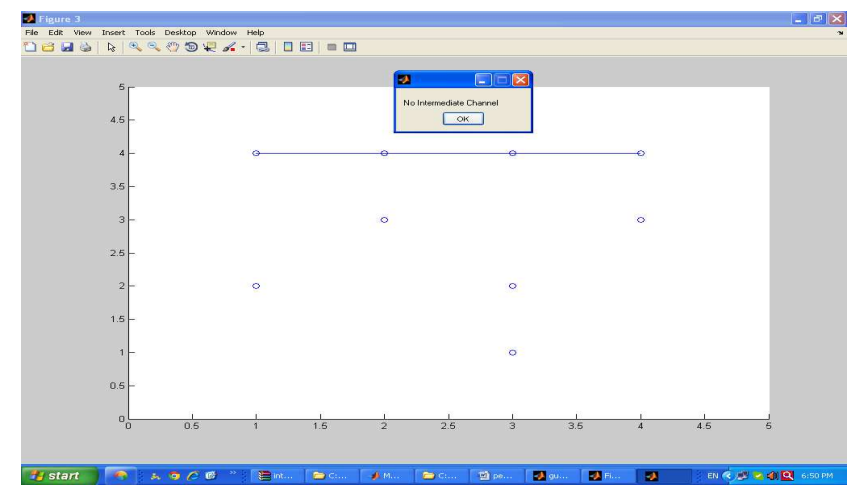

Fig- 5 Path verification from channel created in nodes 
The plot explains about the path created from the design and gives an acknowledgement received from the server.

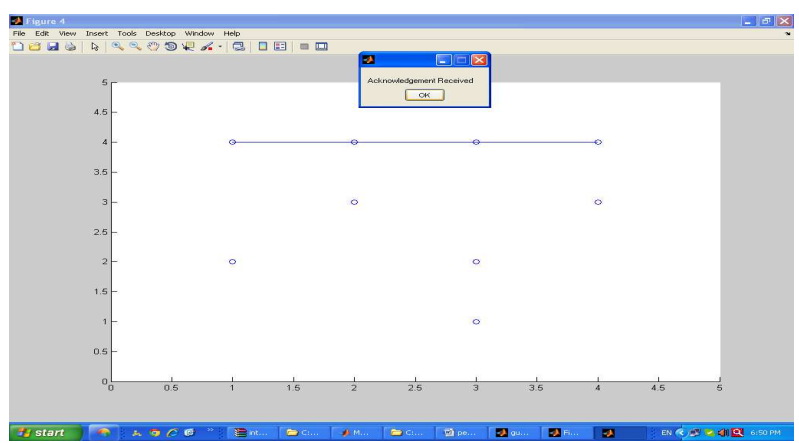

Fig- 6 Acknowledgement received from client

The plot explains about the overall through put with load and obtains the best transmission.

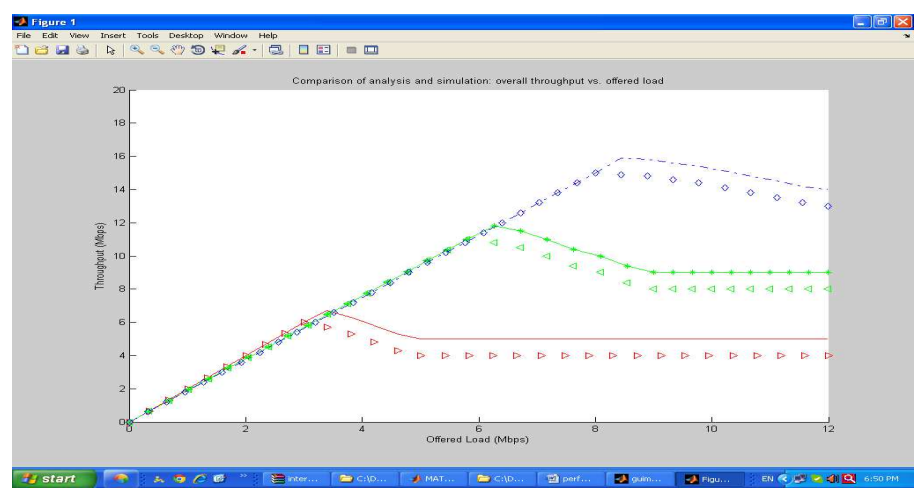

Fig- 7 Overall throughput with data load transmission

The plot explains the status updating of the nodes for the transmission of data.

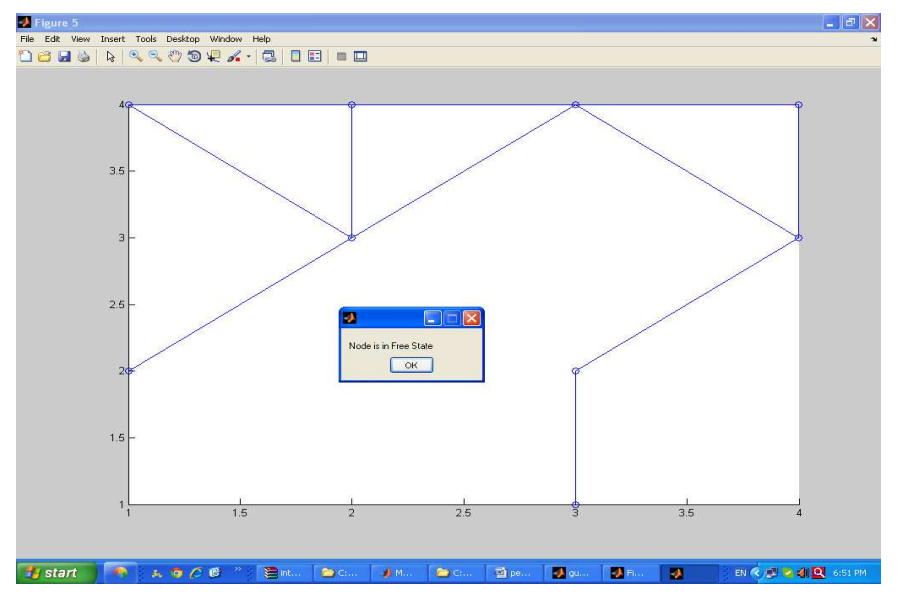

Fig- 8 Status of nodes for data transmission
The plot explains about the traffic checking with any interruption with data transmission in output.

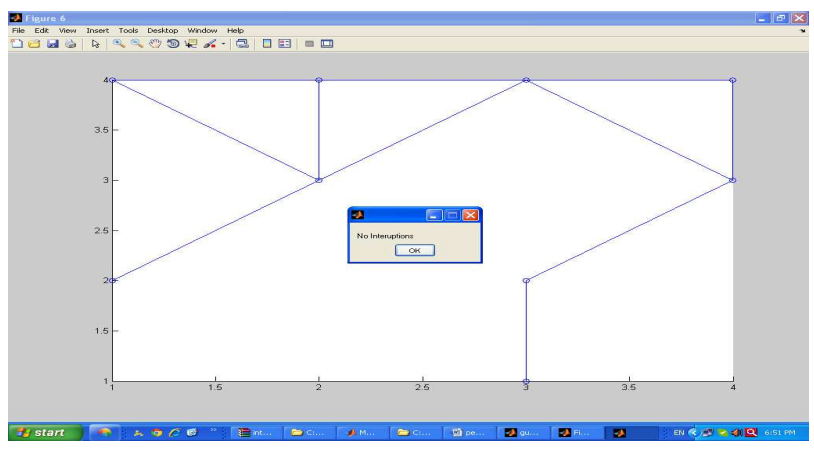

Fig- 9 Traffic checking in data transmission

The plot explains about the data reception from server to client.

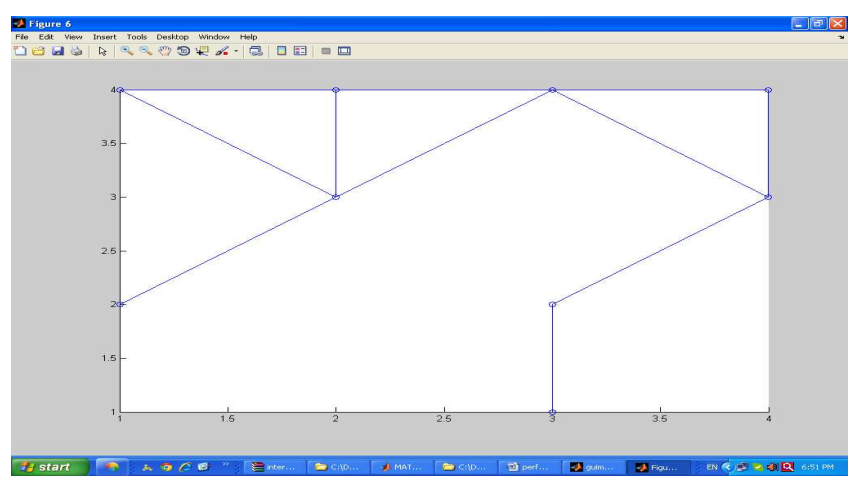

Fig- 10 Data reception from server to client

\section{CONCLUSIONS}

In this paper, we have considered the performance of the TXOP mechanism for multihop wireless networks. We have derived the E2ET and validated the analysis with simulation. From numerical results, we have confirmed that the analytical results agree well with the simulation. Numerical results also showed that a large TXOP limit causes the degradation of the E2ET, and that under a homogeneous offered load condition, setting the TXOP limits of nodes to the same value is effective to achieve high throughput fairness. Note that the analytical model in the paper consists of two parts: a three-node network model and a back off-timer process model. Here, the framelevel queuing behavior is characterized without considering the detailed behavior of the back off timer. This analytical approach was originally developed by Bianchi [7]. From the modeling point of view, our study verified the effectiveness of the analytical framework developed by [7]. This suggests that two-model based approach is significantly useful for performance analysis of IEEE 802.11-based network systems in which frame-level behavior is mainly concerned. 


\section{REFERENCES}

[1] I. F. Akyildiz, X. Wang, and W. Wang, "Wireless mesh networks: a survey," Comput. Netw., vol. 47, no. 4, pp. 445-487, 2005.

[2] IEEE Standard 802.11: Wireless LAN medium access control (MAC) and physical layer (PHY) specifications: medium access control (MAC) quality of service (QoS) enhancements, IEEE 802.11e/D13.0, 2005.

[3] S. Choi, J. Prado, S. Mangold, and S. Shankar, "IEEE 802.11e contention-based channel access (EDCF) performance evaluation," in Proc. IEEE ICC, 2003, pp. 1151-1156.

[4] A. Grilo and M. Nunes, "Performance evaluation of IEEE 802.11e," in Proc. IEEE PIMRC, Sep. 2002, pp. 511-517.

[5] F. Keceli, I. Inan, and E. Ayanoglu, "Fair access provisioning through contention parameter adaptation in the IEEE 802.11e infrastructure basic service set," Arxiv preprint, arXiv:0806.1093, 2008.

[6] T. Suzuki, A. Noguchi, and S. Tasaka, "Effect of TXOP-bursting and transmission error on applicationlevel and user-level QoS in audio-video transmission with IEEE 802.11e EDCA," in Proc. IEEE PIMRC, Sep. 2006, pp. 1-7.

[7] G. Bianchi, "Performance analysis of the IEEE 802.11 distributed coordination function," IEEE J. Sel. Areas Commun., vol. 18, no. 3, 535-48, 2000.

[8] G. Min, J. Hu, W. Jia and M. E. Woodward, "Performance analysis of the TXOP scheme in IEEE 802.11e WLANs with bursty error channels," in Proc. IEEE WCNC, 2009, pp. 1185-1190.

[9] F. Peng, H. M. Alnuweiri, and V. C. M. Leung, "Analysis of burst transmission in IEEE 802.11e wireless LANs," in Proc. IEEE ICC, June 2006, vol. 2, pp. 535-539.

[10] Y. Lee, K. S. Lee, and J. M. Jang, "Saturation throughput analysis of IEEE 802.11e EDCA," Lecture Notes Comput. Science, no. 4682 ,

pp. 1223-1232, 2007.

[11] Y. Gao, D. M. Chiu, and J. C. S. Lui, "Determining the end-to-end throughput capacity in multi-hop networks: methodology and appli-cations," in Proc. Joint International Conf. Measurement Modeling Comput. Syst., June 2006, pp. 39-50.

[12] T. Tainaka, H. Masuyama, S, Kasahara, Y. Takahashi, "A Markovian approach to per-flow throughput unfairness in IEEE 802.11 multihop wireless networks," J. Industrial Management Optimization, vol. 5, no. 3, pp. 493-510, 2009.

[13] R. W. Wolf, "Poisson arrivals see time averages," Operations Research, vol. 30, pp. 223-231, 1982.

[14] D. Vardalis, "On the efficiency and fairness of TCP over wired/wireless networks," Master Thesis, State University of New York at Stony Brook, 2001.
[15] P. T. Giang and K. Nakagawa, "Improvement of fairness by PCRQ scheduling in multihop wireless adHoc networks," in Proc. Asia-Pacific Symp. Queueing Theory Netw. Appl., 2007, pp. 339-348.

\section{BIOGRAPHIES}

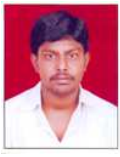

B.Manikanta pursuing M.Tech in specialization of Digital Electronics and communication systems at Chaitanya Engineering college in Department of Electronics and Communication Engineering

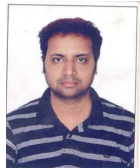

Ch.Sampath Kumar working as an Associate Professor in Chaitanya Engineering college in Department of Electronics and communication Engineering. 